\title{
IMMERSIVE ABSTRACTION: A NEW MORPHOLOGY OF INTUITIVE INTERACTION WITH SYSTEM MODELS
}

\author{
I. Gräßler and J. Pottebaum $\bowtie$ \\ Paderborn University, Germany \\ $\triangle$ jens.pottebaum@hni.upb.de
}

\begin{abstract}
The value of models is well recognised in product and systems engineering. Modelling languages and diagrams are used to capture mental models and to handle model complexity. Literature research indicates that there are only very few approaches to utilise the potential of virtual and augmented reality for supporting tasks in model based (systems) engineering. The paper at hand contributes a new morphology of intuitive interaction for Immersive Abstraction as a holistic approach to extend that coverage. It presents a holistic framework to categorise solutions and future research directions.
\end{abstract}

Keywords: model-based engineering, virtual engineering (VE), 3D modelling, systems engineering (SE), visualisation

\section{Introduction}

The value of models in product design applies for all domains like mechanics (VDI, 2018), software (Völter et al., 2013) and mechatronics (VDI, 2004) as well as for systemic approaches (INCOSE, 2014). Model Based Engineering (MBE) and Model Based Systems Engineering (MBSE) have been introduced as successors of document-centric design (Estefan, 2008). As a key advantage, relationships among engineering artefacts shall be utilized along the entire product design process. Thus, modelling is focused both on single artefacts and relationships. In mechanics and mechatronics, the interconnected representation of requirements, functions, logical structure and physical structure (RFLP) is essential (Kleiner and Kramer, 2013). Every partial model, but especially the interconnected models of systems and sub-systems are extremely large and hard to handle.

Models can be visualised as diagrams using (standard) modelling languages. Figure 1 shows excerpts from a) SysML requirements model, b) BPMN process diagram and c) VOWL ontology representation. The first two examples present abstractions of product resp. system models, focusing on both structure and behaviour of products and systems. SysML (Weilkiens, 2008) extends these two dimensions by the perspective of requirements. The third example extends the scope to metamodels. When concepts of Linked Data (Bizer et al., 2011) and semantic technologies are used, concepts in ontologies have to be used, partially even modelled. VOWL (Visual Notation for OWL Ontologies) is an example for an initiative to enhance visualisation of abstract models (Lohmann et al., 2016).

At the same time, the value of Virtual Prototyping was confirmed in various applications (Leino et al., 2013). The fundaments are built by efficient creation of prototypes and intuitive interaction with them. When envisaging intuitive interaction with data and models, the so called "I3" concept can be applied: 
For Virtual Reality (VR) and Augmented Reality (AR), Burdea and Coiffet (Burdea and Coiffet, 2003) suggest to focus on three dimensions entitled Immersion, Interaction and Imagination. The better engineers immerse themselves into models, the better they can imagine interrelationships and possible effects of changes in a model. For instance, when changing a requirement, they should become aware of the affected system elements that need treatment afterwards (Gräßler et al., 2018).
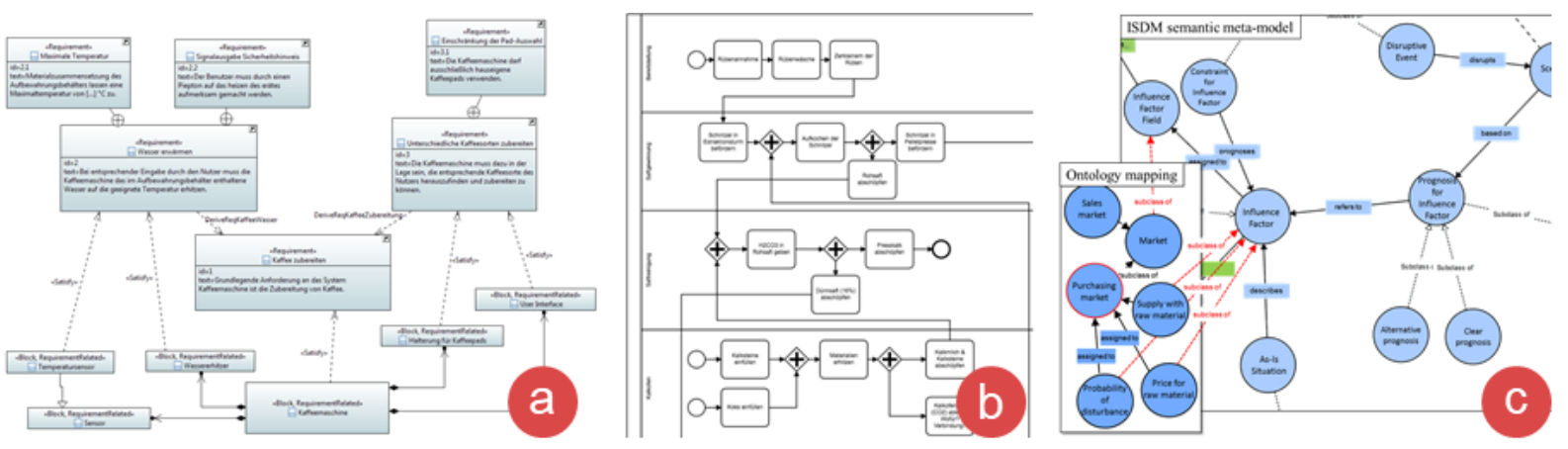

Figure 1. Abstract models requiring immersion: system models, process models and ontologies

The paper at hand is based on the assumption that VR and AR can be used to support (systems) engineers in utilizing the potential of abstract models and their representations in diagrams. Based on fundamentals of both domains (section 2), the research question is elaborated in section 3: How can engineers immerse themselves in system models for more intuitive interaction? A qualitative research approach is applied to analyse related work (section 4) and derive the concept of "Immersive Abstraction" and its core conceptualisation, a morphology of interaction paradigms (section 5). Conclusions are drawn with regard to the morphology and consequent establishment of the concept by future implementations.

\section{Fundamentals}

The research at hand is based on two main considerations: There is a significant challenge in using and especially in maintaining models in product and system design, and at the same time there are technologies for immersive representations to enable intuitive interaction with virtual artefacts.

\subsection{Modelling in product and system design}

Physical structures include geometric characteristics by nature. Thus, they can be represented by Computer Aided Design (CAD). Results of simulations of structural and behavioural characteristics are typically visualised in 3D based on CAD data. The transfer of models into advanced visualisation technologies like Virtual Reality (VR) is supported by CAD exporters resp. VR importers and lightweight data formats. Other types of artefacts like system architectures, requirements catalogues or function structures are mental models of individuals or within design teams (Badke-Schaub et al., 2007). They are typically managed as graphs and formalized by using markup languages. Diagrams are used to visualise views into models. Unified Modelling Language (UML, initially specified for software systems) and Systems Modelling Language (SysML, driven by the Systems Engineering community) are two major examples. In both languages, diagrams can be distinguished with regard to structural and behavioural characteristics of a system; SysML includes requirements as a third type of partial models (Weilkiens, 2008). When creating product-service systems, process models are used to complement system specifications. Therefore, languages like Business Process Modelling Notation (BPMN) are considered (Chinosi and Trombetta, 2012). Visualisation for all of these languages is typically done on 2D planes in special modelling tools without simulation capabilities.

Linkages between partial models are implemented by direct references. Tools for Product Data and Product Lifecycle Management (PDM/PLM) (Stark, 2015) or even Systems Lifecycle Collaboration (see OSLC) (OASIS, 2019) are used to specify such kind of references. As an example, system elements can be linked to requirements by a satisfy relationship. CAD models are specific models detailing elements of a system. Thus, CAD models should be linked on a lower level of the hierarchical system architecture. Practically speaking, this means to add a link from a 2D schematic 
diagram to a CAD model or even a part within a CAD model - and vice versa. Often, vendors of commercially available tools like Dassault (Catia and project management in 3D Experience), Siemens (Teamcenter and Polarion suite) or IBM (Doors) did not close this gap to simplify the interconnection of different types of models. Therefore, finding the correct reference means significant efforts and implies one of the barriers in holistic $\mathrm{MB}(\mathrm{S}) \mathrm{E}$.

While markup languages include syntax for such links between models, semantic technologies can be used to solve this semantic gap. The semantics of model types and their elements can be modelled in ontologies. "An ontology is an explicit specification of a conceptualization." (Gruber, 1993). Its purpose is to be shared within a community. Ontologies include vocabulary, taxonomy and further relationships between concepts. Generic ontologies can be extended by, for instance, domain ontologies (Buchmann and Karagiannis, 2016). Ontologies are never assumed to be "complete", but have to be maintained and extended by corresponding communities. Like other types of meta-models, ontologies are abstract and often large models which are hard to handle. Therefore, there are approaches to simplify visualisation and interaction like the Visual Notation for Web Ontologies (Lohmann et al., 2016). While only few engineers need to deal with extending ontologies or other types of meta-models, they have to be able to identify and select most suitable concepts when annotating technical artefacts along systems engineering and RFLP activities. Therefore, they need intuitive interaction with these kinds of models.

\subsection{Technologies for immersive representations}

Virtual and Augmented Reality applications consist of a VR scene including environment and objects, interaction elements and animations (Burdea and Coiffet, 2003). Additionally, they provide functionality to interact with this scene by various types of input devices.

Augmented Reality (AR) can be used in combination with real-world artefacts. AR can support both representational and constructive use cases. Typical devices for AR are tablet PCs, but also Head Mounted Displays. Three application scenarios are relevant in combination with the use of diagrams:

- Tangible Interaction: physical (standard) elements are created and used as tangible interface dedicated to interaction with models (Billinghurst et al., 2008)

- Hybrid Prototyping (Stark et al., 2009): models are represented both by diagrams and physical prototypes (prototypes are created for engineering purposes, i.e., experiments and reviews)

- Reverse Engineering: models correspond to a product which is already fully or partially developed (Anwer and Mathieu, 2016)

Virtual Reality (VR) adds opportunities for Virtual Engineering (Ovtcharova, 2010) as fourth type of application scenarios. VR does not necessarily mean significant hardware efforts. Even when Head Mounted Displays or CAVEs are not available, displays can be used to visualise virtual environments. Users are represented within the application. They are able to navigate within that scene, trigger events or view animations. Scenes are created by standard 3D modelling tools or specialised VR development kits. Applications are often intended to show close-to-reality environments. Additional objects can be added as 3D objects; 2D graphics or media streams have to be mapped onto planes. Non-3D diagrams have to be converted to an appropriate representation when used in VR. Adopting the concept of model transformation (Czarnecki and Helsen, 2006), options are:

- Texture mapping: diagrams are mapped onto planes or object surfaces

- Element conversion: diagram elements are converted to 2.5D or real 3D objects

- Shape type translation: element types are translated to corresponding 3D representations

- Language extension: markup languages are extended for representational shapes in 3D

While individuals or small groups can implement the upper three options, the fourth option requires community engagement or even standardisation activities.

\section{Methodology}

Research into these two perspectives led to the initial definition of the research question:

How can engineers immerse themselves in system models for more intuitive interaction? 
Related work is identified by literature research (Machi and McEvoy, 2016). It is conducted using combinations of search terms representing the concepts of visualisation (Virtual Reality) and the artefacts to be represented (model, modelling, diagram, SysML, UML, BPMN). The Design Society library and google scholar are used as main sources. All articles are filtered for relevancy. Almost all remaining articles represent reports from single case studies in specific industrial branches. They are qualitatively analysed with regard to a) use cases, b) concepts and c) specificities of case studies. According to the snow ball principle, referenced literature is analysed to expand the knowledge base of related work.

The results are analysed from two different viewpoints: task-oriented and scene-oriented. On the one hand, the goal is to understand how to support design tasks, namely the interaction with models in product design. Tasks mainly subsume the creation, interpretation, manipulation and parameterisation of models. Selected articles are analysed with regard to underlying requirements. On the other hand, there are enablers to enhance imagination, to extend immersion and to facilitate interaction with models. Enablers are mainly data format converters, visualisation concepts and interaction principles. Fundamentals of model transformation (Czarnecki and Helsen, 2006) are used to frame the morphology into an applicable resource for technical implementations (see Figure 2).

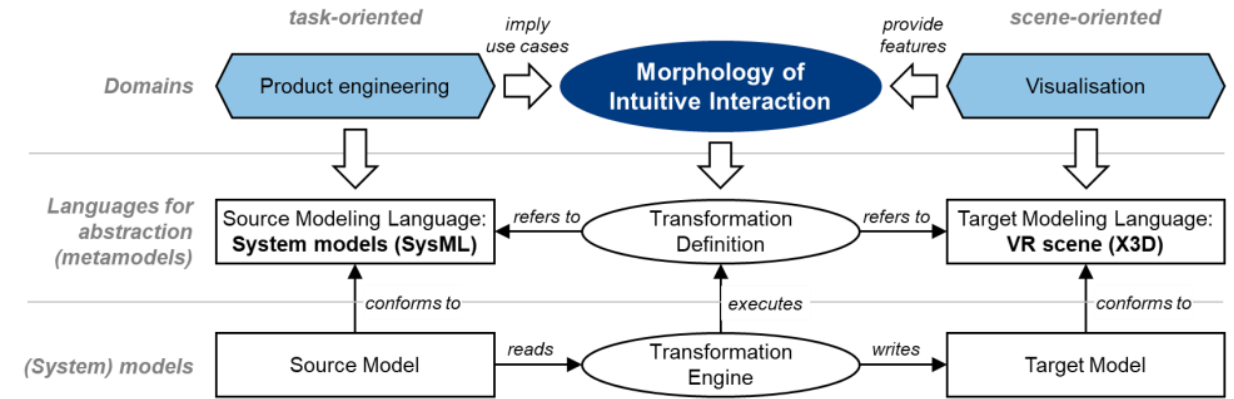

Figure 2. Immersive Abstraction: Transforming system models into VR scenes by applying a morphology of intuitive interaction for Immersive Abstraction

Based on these foundations, a new morphology for intuitive interaction with abstract system models is presented. It is the evolution of cross-domain conceptualisation bridging product engineering tasks and visualisation. The morphology provides a baseline of transformation definitions between system models and VR scenes. It is driven by the concept of design support (Blessing and Chakrabarti, 2009), specifically three complementary perspectives: tasks in product and systems engineering, artefacts of interest within that domain and tools required to perform specific activities fulfilling tasks and interacting with artefacts. These perspectives are correlated with the I3 dimensions of Virtual and Augmented Reality.

\section{Related work}

The Design Society library does not contain any directly related articles. Using combined search terms, there are no indications of authors which are proposing virtual technologies as a tool for creating or maintaining abstract product or system models. Instead, two different lines of applications can be identified: Firstly, approaches that suggest usage of VR for modelling product characteristics like surfaces (Arqueros et al., 2012) or lighting effects (Ekströmer et al., 2019). Secondly, there is a series of three publications by Mahboob et al. They suggest to use SysML for the generation and maintenance of use cases of VR (2017 to 2019, see Mahboob et al., 2019).

Google scholar refers to several papers, but only very few are relevant. There are two categories of articles: some targeting UML modelling in VR, and some focusing on BPMN. SysML modelling is not present so far. Due to the scope of the paper at hand in conceptual modelling, 3D visualisations of software code and its automatic creation was not included in this section.

There are three main approaches with regard to UML modelling in VR. Zhang and Chen (Zhang and Chen, 2005) assume that I3 capabilities of VR support core requirements of conceptual models: understandability, expressiveness, processing independence, checkability and changability. Figure 3 (left screenshot) presents a mockup that they used to investigate the potential of VR with regard to 
sequence diagrams. Gregorovič et al. (2015) suggest a layered representation of class diagrams to reduce complexity in handling them. Their focus in on applying force directed algorithms to optimise the arrangement of the diagram. Weights are applied for possible relationships between classes. Rodrigues et al. (2016) present VisAr3D (Figure 3, in the middle) as a tool that supports automatic creation of 3D UML model representations from UML diagrams. Later publications mainly focus on usage of these models in educational contexts. McIntosh's (2009) main focus was twofold: Firstly, his solution supports conceptual design of embedded software systems. Secondly, he transferred 3D models into an AR application which he entitled '3D UML Mechatronic Diagram' (Figure 3, right). McIntosh provides detailed insights into case studies, for instance, at IBM.
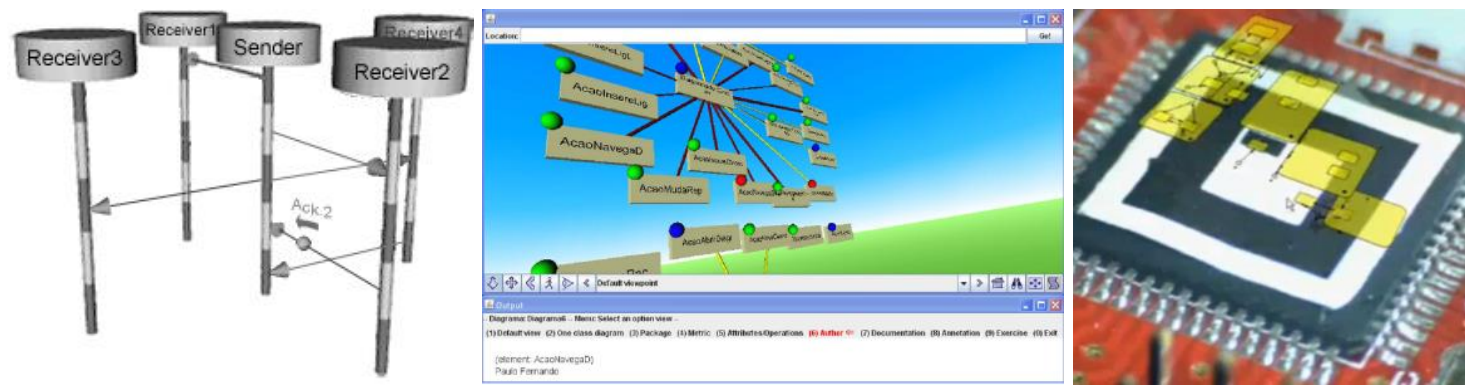

Figure 3. Modelling UML diagrams: scenario on sequence diagrams by Zhang and Chen (2005) (left), automatised generation of 3D content from UML diagrams using VisAr3D (middle) and state machine overlaid on an embedded processor (Mclntosh, 2009) (right)

A second field of applications was identified in (business) process modelling with BPMN. Two teams of researchers published their approaches with complementary objectives. Oberhauser et al. (2018) (see Figure 4) implemented the VR-BPMN tool for "for visualizing, navigating, annotating, and interacting with BPMN models that supports teleportation, fly-through navigation, depicting subprocesses via stacked hyperplanes, drawing annotative associations between BPMN elements, coloring model elements, and tagging textual elements using mixed reality keyboard support". In their paper, they present study results from comparisons of VR-BPMN with paper-based modelling and tool-based BPMN (Camunda process modeler). They extended its application to enterprise architecture models, entitled VR-EA (Oberhauser and Pogolski, 2019). Key use cases are visualisation, navigation and interaction. Brown (2010) builds upon a task analysis leading to nine key requirements for VR-supported process modelling, where "modelling is restricted to high-level conceptual visualisation and communication". His tool is based on Open Simulator and Second Life open source viewer. Key developments are representational shapes for BPMN elements, textures for annotation types, a tool to draw flow connections (see Figure 5, left screenshot), avatar instance animations (like in Figure 5, middle) and annotation functionalities. The modelling use cases are extended by collaboration tools (mind mapper, web browser, slide show delivery and information holodeck) (Brown et al., 2011) (Figure 5, right screenshot). It is then extended to collaborative AR (Poppe et al., 2011).
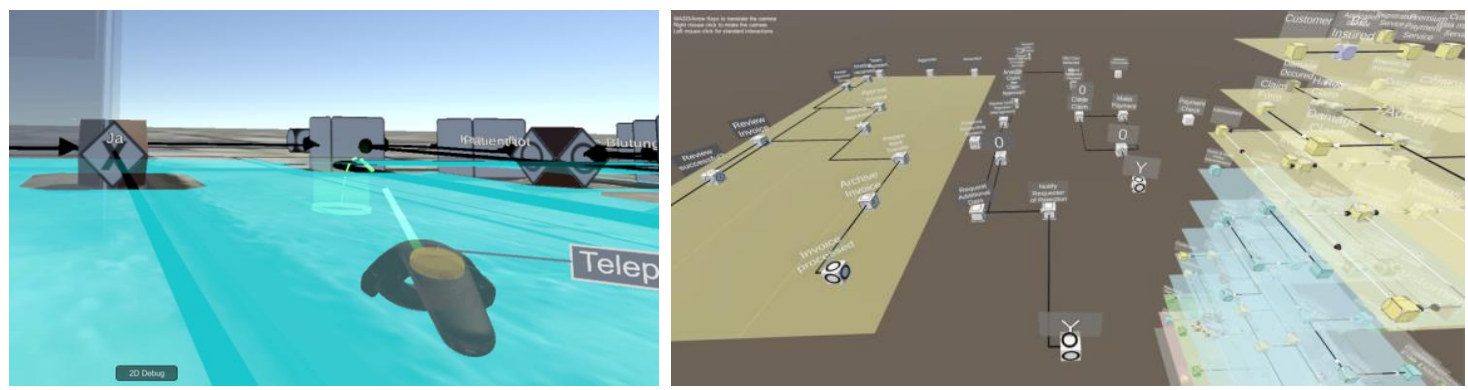

Figure 4. Modelling in VR by Oberhauser et al.: interacting with elements (Oberhauser et al., 2018) (left) and functions for complementary models (Oberhauser and Pogolski, 2019) (right) 

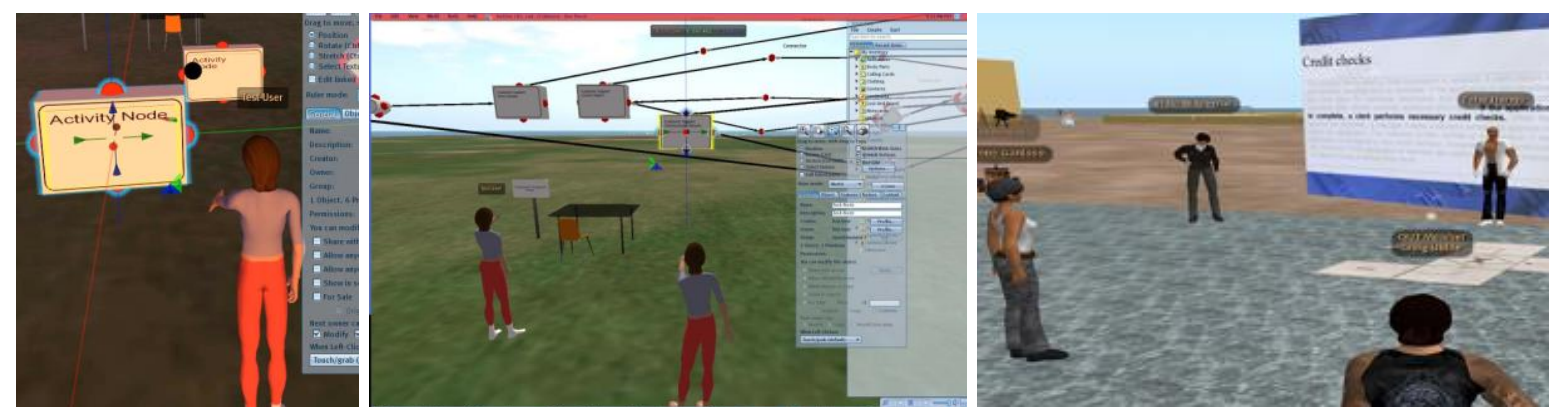

Figure 5. Modelling in VR by Brown et al.: creating and manipulating BPMN models (Brown, 2010) (left/middle), collaborative review (Brown et al., 2011) (right)

\section{Morphology of intuitive interaction for Immersive Abstraction}

The analysis is used to derive requirements of intuitive interaction with system models in virtual environments. Requirements are categorized and complemented by further research influences. The concept is called 'Immersive Abstraction': a new paradigm for designing intuitive modelling tools. Figure 6 presents the three drivers of the morphology:

- Tasks requiring immersion: Tasks along RFLP approaches are correlated with activities to identify categories of immersion requirements. Firstly, these activities are focused on reviewing and verifying models. Secondly, these activities can be categorised to design and analysis of the envisaged product or system. Collaboration is added as a separate category as it a) complements the first four categories and b) requires further functionalities like communication and visualisation of collaborators.

- Artefacts requiring imagination: The approach is focused on models, which are represented by diagrams nowadays. Here, models restricted to single partial SysML models or components models are entitled 'simple models'. 'Complex models' cover cross-domain perspectives, integration of partial models and system models as such. Both simple and complex models can be represented through straightforward texture mapping and in-depth approaches of element conversion (cf. section 2.2). As a second category, augmentation is targeted. Solutions might a) utilise conventional diagrams (for instance, printed on paper) and augment them with virtual content or b) augment real objects like prototypes or products. Hence, tangible interaction, hybrid prototyping and reverse engineering can be implemented.

- Tools enabling interaction: 'tools' subsume elements and operations in virtual environments that enable interaction. Beyond viewing, tools are required to create, to annotate, to manipulate and to parameterise models. While annotation means operations like adding labels or geometric markers, parametrisation means to set parameters or variables or to trigger events, which are specified in static objects like original CAD data or in dynamic models like state machines.

\begin{tabular}{|c|c|c|c|c|c|}
\hline \multirow{2}{*}{$\begin{array}{l}\text { TASKS } \rightarrow \\
\text { Immersion }\end{array}$} & \multicolumn{2}{|c|}{ Model } & \multicolumn{3}{|c|}{ Product/System } \\
\hline & Review & Verify & Design & Analyse & $\begin{array}{l}\text { Colla- } \\
\text { borate }\end{array}$ \\
\hline Requirements & \multicolumn{3}{|c|}{$\begin{array}{c}\text { Descriptions, attributes, priorities, } \\
\text { inter-relationships, } \ldots\end{array}$} & & \multirow{4}{*}{$\begin{array}{c}\text { Define, } \\
\text { measure, } \\
\text { analyse, } \\
\text { improve, } \\
\text { control, } \\
\ldots\end{array}$} \\
\hline Functions & \multicolumn{3}{|c|}{$\begin{array}{c}\text { Descriptions, decompositions, } \\
\text { integration/differentiation, ... }\end{array}$} & & \\
\hline Logical structure & \multicolumn{3}{|c|}{$\begin{array}{l}\text { Sub-systems, components, } \\
\text { disciplines, ... }\end{array}$} & & \\
\hline Physical structure & \multicolumn{3}{|c|}{$\begin{array}{l}\text { Geometries, materials, tolerances, } \\
\text { circuit boards, program code, ... }\end{array}$} & & \\
\hline Validation & & & & $\begin{array}{l}\text { Structure, } \\
\text { behaviour }\end{array}$ & DMAIC \\
\hline Metamodels & \multicolumn{2}{|c|}{$\begin{array}{l}\text { Vocabulary, ontology, } \\
\text { modelling language, ... }\end{array}$} & & $\begin{array}{l}\text { Consistency, } \\
\text { coverage, ... }\end{array}$ & DMAIC \\
\hline
\end{tabular}

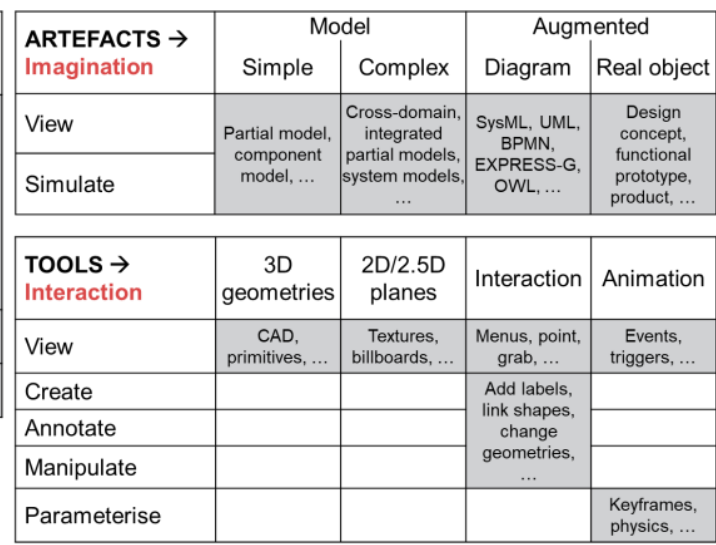

Figure 6. Drivers for utilising 13 dimensions: immersion, imagination and interaction 
Based on these considerations, the holistic morphology is derived. The result is described as a threedimensional cube (Figure 7). The cube is formed by $4 \times 5 \times 6$ building blocks, each of them representing one option to advance I3 dimensions in model based engineering processes: The front plane of the cube shows options for immersion and imagination when simply viewing content, i. e., without interaction. Viewing is extended by four levels of interaction: create models, annotate existing models, manipulate existing models and parameterise behaviour models. Immersion should be targeted through five levels regarding model as subject, product/system as subject and collaboration. Relevant tasks span from reviewing and verifying models through designing and analysing modelled products or systems to model-centred collaboration. While immersion of engineers into an abstract diagram already adds value to their model based work, immersion into the envisaged resp. reversely engineered product/system is the major challenge targeted by the concept of Immersive Abstraction. Therefore, imagination is required. It is achieved by six levels regarding the representation of simple artefacts, complex artefacts to augmentation of models and real world objects. As an example for the latter, a prototype might get augmented by the corresponding system model.

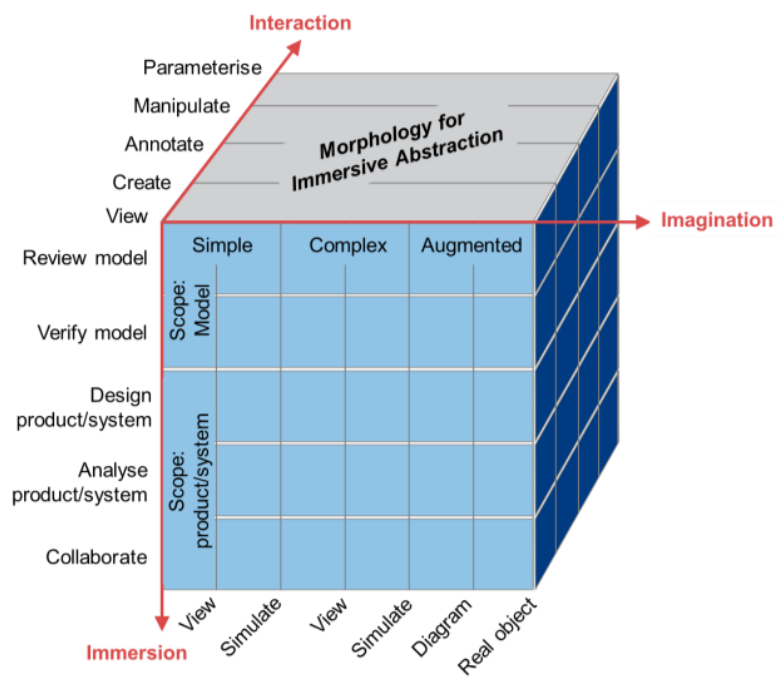

Figure 7. Morphology of intuitive interaction for Immersive Abstraction

The morphology can be applied on three different levels of use cases:

1. single projects focused on a specific product/system to be developed (like shown in Figure 8),

2. team or enterprise wide harmonisation and uptake in engineering processes and

3. (global) standardisation for uptake in product and systems engineering methodologies.

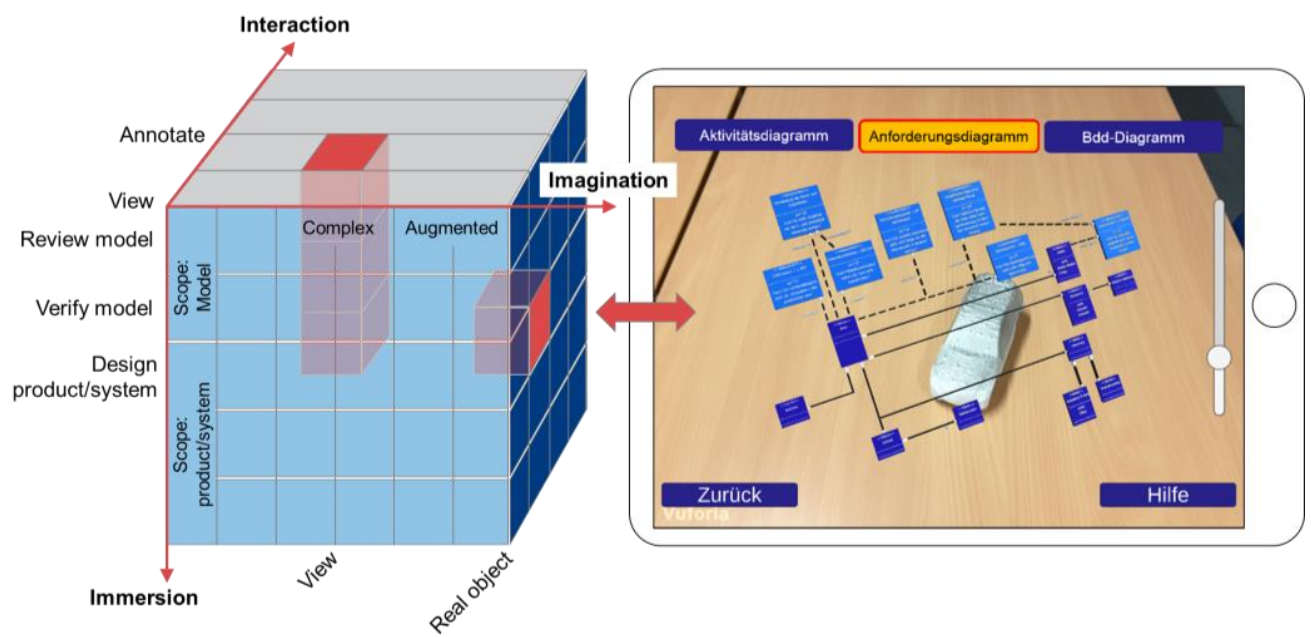

Figure 8. Element conversion applied to SysML diagram combined with augmentation of model and physical prototype (example pre-published in Gräßler et al., 2019) 
In section 2.2, shape type translation and language extension were mentioned as approaches to achieve the third objective. They are related to model transformation levels presented in Figure 2: transformation definition and transformation engine are implemented either within restricted boundaries of an enterprise resp. within the value chain of a product involving OEMs and suppliers, or they are implemented generically as extensions for modelling languages like SysML or BPMN. The latter requires uptake of the morphology as baseline for consensus based processes in communities.

Applicability of the morphology is evaluated in case studies. Figure 8 shows one example: a screenshot of a tablet based AR app supporting review and verification of a SysML requirements model. The application is focused on the design of remote control cars as a mechatronic system; prototyping with 3D printed parts is used in the design phase for customer reviews (for details regarding the application see Gräßler et al., 2019). The requirements model is inter-linked with the block definition diagram (bdd) to visualise logical relationships; abstract blocks are linked with geometries of the augmented prototype of a remote control car. Thus, the application covers four building blocks of the morphology.

\section{Conclusions}

Literature research indicates that there are only very few approaches to utilise the potential of virtual and augmented reality for supporting tasks in model based (systems) engineering. The paper at hand contributes the morphology of intuitive interaction for Immersive Abstraction as a holistic approach to extend that coverage. Firstly, it is based on an analysis of tasks, artefacts and tools in MBE/MBSE. Secondly, it considers immersion, imagination and interaction (I3) as enabling qualities of virtual environments. Both focusing on applications and future research directions, the morphology indicates $4 \times 5 \times 6$ directions to expand ambitions. For each of the building blocks of the morphology, there are options how to implement intuitive interaction with abstract models like system models, process models or meta-models like ontologies. At the same time, the presented results of literature research imply the necessity to investigate applicability and impact for each of these building blocks. Thus, the morphology is a baseline for a broader 'design research roadmap of Immersive Abstraction'. According to basics of model transformation, the transformation definitions have to be prepared and to be agreed upon. Standardisation as part of modelling languages would significantly add value for users, while at the same time paving the ground for the implementation for unified transformation engines. The morphology was created as a contribution to align efforts and to identify synergies of different communities in systems modelling, process modelling, ontology modelling and corresponding visualisation. Future research will, empirically, focus on the significance of options along I3 dimensions and, constructively, focus on gathering and evaluating solutions for specific building blocks.

\section{References}

Anwer, N. and Mathieu, L. (2016), "From reverse engineering to shape engineering in mechanical design", CIRP Annals, Vol. 65 No. 1, pp. 165-168. https://doi.org/10.1016/j.cirp.2016.04.052

Arqueros, N.J., Prieto, P.A. and Zúñiga, M.D. (2012), "A new tool for immersive 3D free-form surface modelling and refining", in Marjanović, D. (Ed.), Design 2012: Proceedings of the 12th International Design Conference, Dubrovnik, 21-24 May 2012, Design Society, pp. 365-372.

Badke-Schaub, P. et al. (2007), "Mental models in design teams: a valid approach to performance in design collaboration?", CoDesign, Vol. 3 No. 1, pp. 5-20.

Billinghurst, M., Kato, H. and Poupyrev, I. (2008), "Tangible augmented reality", in Hart, J.C. (Ed.), ACM SIGGRAPH Asia 2008: Special Interest Group on Computer Graphics and Interactive Techniques Conference, Singapore, ACM Press, New York, New York, USA, pp. 1-10. https://doi.org/10.1145/1508044.1508051

Bizer, C., Heath, T. and Berners-Lee, T. (2011), "Linked Data", in Sheth, A. (Ed.), Semantic services, interoperability, and web applications: Emerging concepts, Vol. 284, IGI Global, Hershey, pp. 205-227.

Blessing, L.T.M. and Chakrabarti, A. (2009), DRM, a Design Research Methodology, Springer London, London.

Brown, R.A. (2010), "Conceptual modelling in 3D virtual worlds for process communication", in Proceedings of the Seventh Asia-Pacific Conference on Conceptual Modelling (APCCM 2010), Brisbane, Australia, Australian Computer Society, Darlington, pp. 25-32.

Brown, R.A., Recker, J. and West, S. (2011), "Using virtual worlds for collaborative business process modeling", Business Process Management Journal, Vol. 17 No. 3, pp. 546-564. https://doi.org/10.1108/ 14637151111136414 
Buchmann, R.A. and Karagiannis, D. (2016), "Enriching Linked Data with Semantics from Domain-Specific Diagrammatic Models", Business \& Information Systems Engineering, Vol. 58 No. 5, pp. 341-353.

Burdea, G.C. and Coiffet, P. (2003), Virtual Reality Technology, Wiley - IEEE Ser, 2nd ed., John Wiley \& Sons Incorporated, New York.

Chinosi, M. and Trombetta, A. (2012), "BPMN: An introduction to the standard", Computer Standards \& Interfaces, Vol. 34 No. 1, pp. 124-134. https://doi.org/10.1016/j.csi.2011.06.002

Czarnecki, K. and Helsen, S. (2006), "Feature-based survey of model transformation approaches", IBM Systems Journal, Vol. 45 No. 3, pp. 621-645. https://doi.org/10.1147/sj.453.0621

Ekströmer, P. et al. (2019), "Shedding Light on Game Engines and Virtual Reality for Design Ideation", in Wartzack, S., Schleich, B., Guerreiro Gonçalves, M., Eisenbart, B. and Völkl, H. (Eds.), Responsible Design for Our Future: Proceedings of the 22nd International Conference on Engineering Design (ICED19), Delft, 5-8 August 2019, Cambridge University Press, Cambridge, pp. 2003-2010.

Estefan, J.A. (2008), Survey of Model-Based Systems Engineering (MBSE) Methodologies, available at: http://www.omgsysml.org/MBSE_Methodology_Survey_RevB.pdf (accessed 9 July 2019).

Gräßler, I., Oleff, C. and Taplick, P. (2019), “Augmented Reality für die Vermittlung von Systems EngineeringKompetenzen”, in Bertram, T., Corves, B., Gräßler, I. and Janschek, K. (Eds.), Tagungsband der Fachtagung Mechatronik 2019, Paderborn, 27-28 March 2019, pp. 180-185. https://doi.org/10.17619/UNIPB/1-770

Gräßler, I. et al. (2018), "Semi-Automatized Assessment of Requirement Interrelations", in Marjanović, D., Štorga, M., Škec, S., Bojčetić, N. and Pavković, N. (Eds.), Design 2018: Proceedings of the 15th International Design Conference, Dubrovnik, Croatia, 21-24 May 2018, Design Society, pp. 325-334. https://doi.org/10.21278/idc.2018.0298

Gregorovič, L., Polasek, I. and Sobota, B. (2015), "Software Model Creation with Multidimensional UML", in Khalil, I. (Ed.), Information and communication technology: Third IFIP TC 5/8 international conference, ICT-EurAsia 2015, and 9th IFIP WG 8.9 working conference, CONFENIS 2015, held as part of WCC 2015, Daejeon, Korea, October 4-7, 2015 proceedings, Lecture Notes in Computer Science, Vol. 9357, Springer, Cham, pp. 343-352. https://doi.org/10.1007/978-3-319-24315-3_35

Gruber, T.R. (1993), “A translation approach to portable ontology specifications”, Knowledge acquisition, Vol. 5 No. 2, pp. 199-220.

INCOSE (2014), Systems Engineering Handbook: A Guide for System Life Cycle Processes and Activities, San Diego.

Kleiner, S. and Kramer, C. (2013), "Model Based Design with Systems Engineering Based on RFLP Using V6", in Abramovici, M. and Stark, R. (Eds.), Smart Product Engineering: Proceedings of the 23rd CIRP Design Conference, Bochum, 11-13 March 2013, Springer, Berlin, Heidelberg, pp. 93-102.

Leino, S.-P.S., Koivisto, T. and Riitahuhta, A. (2013), "Value of virtual prototyping - A strategic resource based view”, in Lindemann, U., Srinivasan, V., Kim, Y.S., Lee, S.W., Clarkson, J. and Cascini, G. (Eds.), Design for harmonies: Proceedings of the 19th International Conference on Engineering Design (ICED13), Seoul, Korea, 19-22 August 2013, Design Society, Castle Cary, Somerset, pp. 249-258.

Lohmann, S. et al. (2016), "Visualizing ontologies with VOWL", Semantic Web, Vol. 7 No. 4, pp. $399-419$. https://doi.org/10.3233/SW-150200

Machi, L.A. and McEvoy, B.T. (2016), The literature review: Six steps to success, Third Edition, Corwin, Thousand Oaks, California.

Mahboob, A. et al. (2019), "The Reuse of SysML Behaviour Models for Creating Product Use Cases in Virtual Reality", in Wartzack, S., Schleich, B., Guerreiro Gonçalves, M., Eisenbart, B. and Völkl, H. (Eds.), Responsible Design for Our Future: Proceedings of the 22nd International Conference on Engineering Design (ICED19), Delft, 5-8 August 2019, Cambridge University Press, Cambridge, pp. 2021-2030. https://doi.org/10.1017/dsi.2019.208

McIntosh, P.M. (2009), "X3D-UML: User-Centred Design, Implementation and Evaluation of 3D UMLUsing X3D”, Dissertation, School of Computer Science and Information Technology, RMIT University, Melbourne, Australia.

OASIS (2019), Open Services for Lifecycle Collaboration, available at: https://open-services.net/ (accessed 24 November 2019).

Oberhauser, R. and Pogolski, C. (2019), "VR-EA: Virtual Reality Visualization of Enterprise Architecture Models with ArchiMate and BPMN", in Shishkov, B. (Ed.), Business Modeling and Software Design: 9th International Symposium, BMSD 2019, Lisbon, Portugal, July 1-3, 2019, Proceedings, Lecture Notes in Business Information Processing, Vol. 356, 1st ed., Springer International Publishing; Imprint; Springer, Cham, pp. 170-187. https://doi.org/10.1007/978-3-030-24854-3_11

Oberhauser, R., Pogolski, C. and Matic, A. (2018), "VR-BPMN: Visualizing BPMN Models in Virtual Reality", in Shishkov, B. (Ed.), Business Modeling and Software Design: 8th International Symposium, BMSD 2018, 
Vienna, Austria, July 2-4, 2018, Proceedings, Lecture Notes in Business Information Processing, Vol. 319, Springer International Publishing, Cham, pp. 83-97. https://doi.org/10.1007/978-3-319-94214-8_6

Ovtcharova, J. (2010), "Virtual Engineering: Principles, Methods and Applications", in Marjanović, D. (Ed.), Design 2010: Proceedings of the 11th International Design Conference, Dubrovnik, Croatia, 17-20 May 2010, Design Society, pp. 1267-1274.

Poppe, E. et al. (2011), “A prototype augmented reality collaborative process modelling tool”, in Rinderle-Ma, S., Toumani, F. and Wolf, K. (Eds.), Business process management: 9th international conference (BPM 2011), Clermont-Ferrand, France, 28 August-2 September 2011, Springer, Berlin.

Rodrigues, C.S.C. et al. (2016), "VisAr3D: An Innovative 3D Visualization of UML Models", in Dillon, L., Visser, W. and Williams, L. (Eds.), Proceedings of the 38th International Conference on Software Engineering Companion - ICSE '16, Austin, Texas, 14 May 2016-22 May 2016, ACM Press, New York, New York, USA, pp. 451-460. https://doi.org/10.1145/2889160.2889199

Stark, J. (2015), Product Lifecycle Management: Volume 1: 21st Century Paradigm for Product Realisation, Decision Engineering, 3rd ed., Springer International Publishing, Cham.

Stark, R. et al. (2009), "Smart Hybrid Prototyping zur multimodalen Erlebbarkeit virtueller Prototypen innerhalb der Produktentstehung", in Lichtenstein, A. (Ed.), Der Mensch im Mittelpunkt technischer Systeme: 8. Berliner Werkstatt Mensch-Maschine-Systeme, Berlin, 7-9 October 2009, VDI-Verlag, Düsseldorf.

VDI (2004), VDI 2206 - Design methodology for mechatronic systems No. 2206, Verein Deutscher Ingenieure, Düsseldorf, available at: www.vdi.de.

VDI (2018), VDI 2221 Entwicklung technischer Produkte und Systeme - Modell der Produktentwicklung No. VDI 2221, Verein Deutscher Ingenieure, Düsseldorf.

Völter, M. et al. (2013), Model-Driven Software Development: Technology, Engineering, Management, Wiley Software Patterns Series, Wiley.

Weilkiens, T. (2008), Systems Engineering with SysML/UML: Modeling, Analysis, Design, The MK / OMG Press, 1. Aufl., Elsevier professional, s.1.

Zhang, B. and Chen, Y.-S. (2005), "Enhancing UML Conceptual Modeling through the Use of Virtual Reality", in Sprague, R.H. (Ed.), Proceedings of the 38th Annual Hawaii International Conference on System Sciences, Big Island, HI, USA, 3-6 January 2005, IEEE Computer Society Press, Los Alamitos, California. 